\title{
B.A. Akbarnia, M. Yazici, G. Thompson (eds): The growing spine
}

\author{
Springer Verlag, Heidelberg, 2010, 656 pp, 160 figs, 27 tabs, hard cover, \\ ISBN: 978-3-540-85206-3, USD 239,00
}

\section{Concezio Di Rocco}

Received: 15 March 2011 / Accepted: 15 March 2011 / Published online: 8 April 2011

(C) Springer-Verlag 2011

While spine surgery has become a very important field of neurosurgeons' practice in recent years, the pediatric neurosurgeon still appears to be relatively reluctant in engaging her/ himself in the treatment of congenital and developmental spinal disorders. The phenomenon may be explained by various factors but the most important of them is surely the conviction that only a few congenital or acquired pediatric pathological anomalies of the spine require the neurosurgical expertise, with the possible exception of the repair of congenital dysraphic lesions. Such a conviction is certainly challenged by this excellent multiauthored book edited by B. A. Akbarnia, M. Yazici, and G.H. Thompson, which illustrates the whole spectrum of the pediatric disorders which may affect the growth of the spine, from the neonatal period to adolescence. Actually, the book covers all the aspects which characterize the growing spine, from its embryologic development, biomechanical characteristics to the changes that may be induced by trauma, tumors, metabolic diseases, dysraphisms, neurofibromatosis, and neuromuscular diseases. Scoliosis, spondylolisthesis, and spine deformities are described in details together with the diagnostic work up and the related surgical and orthotic treatments.

Altogether, the 50 chapters which make up the volume, all of them written in a concise and extremely didactic style, represent a unique reference source for the pediatric neurosurgeon willing to explore the field of spinal surgery and to become an important member of a team which comprises the pediatrician, the pediatric orthopaedist, the neurologist, as well as the specialized neuroradiologist, anaesthesiologist, physiotherapist, and nursing personnel. Indeed, devoted chapters of the book aim at illustrating the role exerted by these specialists in the different phases of the normal and pathological growth of the spine.

By reading the text, the pediatric neurosurgeon will become acquainted with a variety of conditions which potentially could benefit of a neurosurgical contribution, consequently justifying a specific interest by the pediatric neurosurgeon. It is worth to note that out of the 50 chapters of the book, only one has been written by a pediatric neurosurgeon. Namely, the one concerning myelomeningocele repair. Then, let us hope that, as a direct effect of the book itself, a second edition of it may find a larger proportion of chapters written by pediatric neurosurgeons! In summary, this high quality and comprehensive volume is a timely publication which should influence the attitude of pediatric neurosurgeon in favor of a major involvement in the treatment of the growing spine disorders.

The editors should be complimented for having been able to gather an excellent group of specialists and having maintained an uniformously high standard for all the different contributions coming from the various authors. As usual, the publisher Springer has contributed with its care, quality of printing, and graphic architecture to make the book very readable and appealing.
C. Di Rocco $(\bowtie)$

Department of Neurosurgery, Policlinico Gemelli,

Rome, Italy

e-mail: cdirocco@rm.unicatt.it 